\title{
Analysis of the dynamic behavior of composite plates subjected to impact
}

\author{
Mohamad Abdel Wahab ${ }^{1, a}$, Tony Jabbour ${ }^{2}$ and Farouk El-Dahabi ${ }^{2}$ \\ 1 Faculty of Engineering 1, Lebanese University, Tripoli, Lebanon \\ 2 ISSAE-Cnam Liban, Lebanese University, Beirut, Lebanon
}

Received 2 May 2015, Accepted 10 February 2016

\begin{abstract}
This paper presents a simple analytical model of the dynamic response of a structure subjected to a low speed impact, in order to predict global damage. The model is based on thick plate theory developed by Reddy and takes into account the through thickness shear. An indentation law based on the theory of Hertz is determined experimentally to model the local response. A numerical integration method is used to calculate the contact force versus time, by combining the indentation with the dynamic response of the structure. The validation has been performed by comparing results with measurements obtained on a falling weight set-up, and a satisfactory correlation is obtained. This model leads to the identification of parameters inducing damage. The modal damping coefficient is taken into account.
\end{abstract}

Key words: Composite / impact / dynamic response / thick plate / damping

\section{Introduction}

The impact is one of the main and most damaging causes of laminated materials (monolithic or sandwiches). The damage created by an impact can significantly reduce stiffness of a multilayered structure, especially in compression mode and bending. It has been observed that when composite plates are subjected to impact loading, two types of damage need to be considered: local damage involving crushing beneath the impactor, and global damage revealed by cracks due to flexure of the plate. Various experimental studies, performed on a wide range of materials, have described these different types of damage $[1,2]$. For composite materials laminated panel type or sandwich, internal induced damage may become severe and weaken the structure. The effect of the low-speed impact is therefore a threat to the safety of composite structures.

In order to study these different types of damage, which depend on both the lay-up and material properties of the constituents and the boundary conditions, finite element (FE) methods, can be applied [3-6].

However, the dynamic response model of a thick composite plate subjected to impact loading can also be studied using analytical expressions [7], which can enable rapid parametric studies to be performed without the numerical difficulties and complexity of FE models. In this paper an analytical approach has been developed which

${ }^{a}$ Corresponding author: abdulwahabmo1@gmail.com accounts for the structural aspects of the panel and its behavior during localized loading under the impactor.

The behaviour of laminated composite plates subsequent to impact is a subject which, today, attracts the interest of many researchers. There are numerous published articles on the subject; however, numerical simulations using the finite element method remain a favourite approach to the problem which can often involve complex models. A simple dynamical method has been used in this work, based on modal superposition. This study aims at analysing the global displacement modes arising from overloads occurring at locations away from the impacted zones. This should facilitate the preliminary design of composite plates without having to utilize costly numerical methods.

The dynamical response is obtained by modal superposition, the modes being calculated analytically assuming a simple mathematical model of the thick plate vibrating under simple boundary conditions. The plate model is based on the work of Reddy [8] and includes transverse shear effects. The displacement field can then be calculated at any point of the plate.

In order to calculate the contact force due to impact, it is necessary to formulate an indentation law to model the localized effects of the shock. In this paper, a law of the Hertzian type has been adopted and verified experimentally. 


\section{List of symbols}

\begin{tabular}{|ll|}
\hline$a, b$ & dimensions of the plate \\
$A_{i j}$ & membrane rigidity \\
$B_{i j}$ & coupling rigidity (membrane-bending) \\
$D_{i j}$ & shear-bending stiffness \\
$E_{i}, E$ & Young modulus \\
$F(t)$ & contact force \\
$F_{i}$ & value of the force during an interval of time \\
$g$ & acceleration of gravity \\
$G_{i j}, G$ & shear modulus \\
$I_{z}, I$ & moment of inertia \\
$k_{z}$ & Hertzian coefficient \\
$L$ & Lagrange \\
$M_{n m}$ & generalized mass of mode $n, m$ \\
$m_{o}$ & mass of the impactor \\
$p$ & transversal load \\
$R$ & radius of the impactor \\
$s(t)$ & displacement of the impactor \\
$T$ & contact time \\
$U_{d}$ & energy of deformation \\
$u, v$ & displacements of the mid plane \\
$V_{d}$ & potential energy of the structure \\
$V_{0}$ & velocity of the impactor before impact \\
$w$ & transverse displacement of the structure \\
$\alpha$ & penetration \\
$\xi_{n m}$ & generalized damping coefficient of the mode $n, m$ \\
$\varphi_{x}, \varphi_{y}$ & rotations of the normal \\
$\sigma_{i j}$ & stresses \\
$\varepsilon_{i j}$ & strains \\
$\nu_{i j}$ & Poisson's ratio \\
$\rho$ & density \\
$h$ & thickness \\
\hline &
\end{tabular}

A numerical scheme makes it possible to integrate, with respect to time, the two mechanical phenomena, the local deformation and the response of the structure.

To validate the model, low energy impact tests were performed by dropping a rigid sphere of mass $10.9 \mathrm{~kg}$ onto composite sandwich panels at various impacting velocities. Laminates, formed of orthotropic symmetrical layers, are composite materials with long fibers and an organic matrix. A parametric study was then performed to study the influence of the dimensions of the plate and the mechanical properties of the material on the dynamic response.

\section{Theory of thick laminated plates}

To study the vibrational motion of a thick composite structure, the elements of the theory of thick laminated plates needed to study the dynamic behavior are briefly presented.

The fundamental assumptions of the theory of thick plates are [9]:

- the plates are composed of a stack of parallel layers;

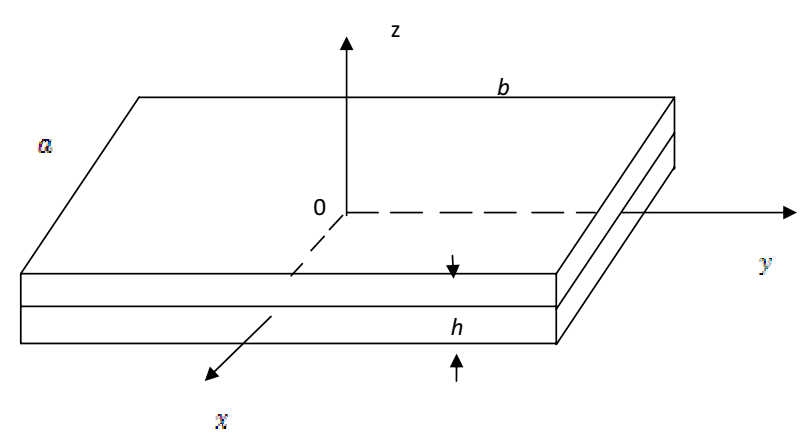

Fig. 1. Schematic of a thick monolithic plate.

- the thickness $\mathrm{h}$ is greater than $1 / 25$ th of the smaller side a (see Fig. 1);

- the elastic behavior for each layer is linear;

- the shear stress is significant;

- the displacements and deformations are small.

The stresses $\sigma_{i j}$ must satisfy the following conditions on the upper and lower sides of the plate:

$$
\begin{aligned}
\sigma_{x z}\left(x, y, \pm \frac{h}{2}, t\right) & =\sigma_{y z}\left(x, y, \pm \frac{h}{2}, t\right)=0 \\
\sigma_{z z}\left(x, y, \frac{h}{2}, t\right) & =p^{+}(x, y, t) \\
\sigma_{z z}\left(x, y,-\frac{h}{2}, t\right) & =p^{-}(x, y, t)
\end{aligned}
$$

where $p^{ \pm}(x, y, t)$ is the transverse load applied on the upper and lower faces of the structure.

In the case of a monolithic thick plate, according to Reddy [8], the displacement field has the following kinematics:

$$
\left\{\begin{aligned}
u(x, y, z, t)= & u^{0}(x, y, t)+z \varphi_{x}(x, y, t)+z^{2} \theta_{x}(x, y, t) \\
& +z^{3} \psi_{x}(x, y, t) \\
v(x, y, z, t)= & v^{0}(x, y, t)+z \varphi_{y}(x, y, t)+z^{2} \theta_{y}(x, y, t) \\
& +z^{3} \psi_{y}(x, y, t) \\
w(x, y, z, t)= & w(x, y, t)
\end{aligned}\right.
$$

where $u^{0}, v^{0}$ and $w$ are, respectively, the displacements in the median plane and the transverse displacement of the structure, $z$ is the vertical coordinate.

$\varphi_{x}$ and $\varphi_{y}$ are the components of the normal to the median plane along the axes $x$ and $y$ respectively. The functions $\theta_{x}, \theta_{y}, \psi_{x}$, and $\psi_{y}$ can be determined by using conditions on the transverse shear stresses.

It is important at this point to recall that, according to Equation (1), the conditions on the constraints that need to be checked on the upper and lower sides of the plate, imply, thanks to the linear relationship between stress and strain, that the functions listed above have the 
following form:

$$
\begin{aligned}
\theta_{x} & =0, \theta_{y}=0, \psi_{x}=-\frac{4}{3 h^{2}}\left(\frac{\partial w}{\partial x}+\phi_{x}\right) \\
\text { and } \quad \psi_{y} & =-\frac{4}{3 h^{2}}\left(\frac{\partial w}{\partial y}+\phi_{y}\right)
\end{aligned}
$$

Substituting Equation (4) in the Equation (3), we obtain the form used for the displacement field:

$$
\left\{\begin{array}{l}
u(x, y, z, t)=u^{0}(x, y, t)+z \phi_{x}(x, y, t)-\frac{4 z^{3}}{3 h^{2}}\left(\frac{\partial w}{\partial x}+\phi_{x}\right) \\
v(x, y, z, t)=v^{0}(x, y, t)+z \phi_{y}(x, y, t)-\frac{4 z^{3}}{3 h^{2}}\left(\frac{\partial w}{\partial y}+\phi_{y}\right) \\
w(x, y, z, t)=w(x, y, t)
\end{array}\right.
$$

\subsection{Strain field}

The strain field is deduced from the displacement field in Equation (5), thus we obtain

$$
\left\{\begin{aligned}
\varepsilon_{x x}= & u_{, x}^{0}+z \varphi_{x, x}-\frac{4 z^{3}}{3 h^{2}}\left(\frac{\partial^{2} w}{\partial x^{2}}+\varphi_{x, x}\right) \\
\varepsilon_{y y}= & v^{0}{ }_{, y}+z \varphi_{y, y}-\frac{4 z^{3}}{3 h^{2}}\left(\frac{\partial^{2} w}{\partial y^{2}}+\varphi_{y, y}\right) \\
2 \varepsilon_{x z}= & \left(\varphi_{x}+\frac{\partial w}{\partial x}\right)\left(1-\frac{4 z^{2}}{h^{2}}\right) \\
2 \varepsilon_{y z}= & \left(\varphi_{y}+\frac{\partial w}{\partial y}\right)\left(1-\frac{4 z^{2}}{h^{2}}\right) \\
2 \varepsilon_{x y}= & u_{, y}^{0}+v_{, x}^{0}+z\left(\varphi_{x, y}+\varphi_{y, x}\right) \\
& -\frac{4 z^{3}}{3 h^{2}}\left(2 \frac{\partial^{2} w}{\partial x \partial y}+\varphi_{x, y}+\varphi_{y, x}\right)
\end{aligned}\right.
$$

\subsection{Stress field}

The behavior law for thick plates is expressed using the following equation:

$$
\left\{\begin{array}{l}
\sigma_{x x} \\
\sigma_{y y} \\
\sigma_{y z} \\
\sigma_{x z} \\
\sigma_{x y}
\end{array}\right\}=\left[\begin{array}{lllll}
Q_{11} & Q_{12} & 0 & 0 & Q_{16} \\
Q_{12} & Q_{22} & 0 & 0 & Q_{26} \\
0 & 0 & Q_{44} & Q_{45} & 0 \\
0 & 0 & Q_{54} & Q_{55} & 0 \\
Q_{16} & Q_{26} & 0 & 0 & Q_{66}
\end{array}\right]\left\{\begin{array}{l}
\varepsilon_{x x} \\
\varepsilon_{y y} \\
\varepsilon_{y z} \\
\varepsilon_{x z} \\
\varepsilon_{x y}
\end{array}\right\}+\sigma_{z z}\left\{\begin{array}{l}
R_{1} \\
R_{2} \\
0 \\
0 \\
0
\end{array}\right\}
$$

where the coefficients of stiffness of a thick laminate composed of $n$ layers of the same properties are expressed as follows:

$$
R_{i}=\frac{C_{i 3}}{C_{33}} \text { for } i=1,2
$$

where $C_{i j}$ are the stiffness coefficients of the material.

and

$$
Q_{i j}=C_{i j}-\frac{C_{i 3} C_{3 j}}{C_{33}} \quad \text { if } i, j=1,2,6
$$

$$
Q_{i j}=C_{i j} \text { if } i, j=4,5
$$

These coefficients can be expressed if the layers have the direction of the principal axis:

$$
\begin{array}{lccc}
Q_{11}=\frac{E_{1}}{\Omega} & Q_{22}=\frac{E_{2}}{\Omega} & Q_{12}=\frac{\nu_{12} E_{2}}{\Omega} & \Omega=1-\nu_{12} \nu_{21} \\
Q_{44}=G_{23} & Q_{55}=G_{13} & Q_{66}=G_{12} \\
R_{1}=\frac{E_{1}}{E_{3}} \frac{\left(\nu_{31}+\nu_{12} \nu_{32}\right)}{\Omega} & R_{2}=\frac{E_{2}}{E_{3}} \frac{\left(\nu_{32}+\nu_{12} \nu_{31}\right)}{\Omega}
\end{array}
$$

\subsection{Fundamental relations of the behavior of a laminate taking into account the transverse shear}

In the case of a thick plate, the fundamental equation of dynamic can be written as follows $\operatorname{div} \sigma=\rho \ddot{U}$. Thus we obtain a new system of equations

$$
\left\{\begin{array}{l}
M_{x, x}+M_{x y, y}-Q_{x}=f_{u} \\
M_{x y, x}+M_{y, y}-Q_{y}=f_{v} \\
Q_{x, x}+Q_{y, y}-P_{w}=f_{w}
\end{array}\right.
$$

where $p_{W}=\left[\sigma_{z z}\right]_{-h / 2}^{h / 2}$

$$
\begin{aligned}
\left(M_{x}, M_{y}, M_{x y}\right) & =\int_{-h / 2}^{h / 2} z\left(\sigma_{x x}, \sigma_{y y}, \sigma_{x y}\right) \mathrm{d} z \\
\left(Q_{x}, Q_{y}\right) & =\int_{-h / 2}^{h / 2}\left(\sigma_{x z}, \sigma_{y z}\right) \mathrm{d} z
\end{aligned}
$$

and

$$
\left(\left(f_{u}, f_{v}\right)=\int_{-h / 2}^{h / 2} \rho z(\ddot{u}, \ddot{v}) \mathrm{d} z\right), \quad\left(f_{w}=\int_{-h / 2}^{h / 2} \rho \ddot{w} \mathrm{~d} z\right)
$$

\section{Analytical method}

From the theory of thick plates, we can establish an analytical method for the study of the dynamic response of a thick monolithic plate subjected to a low impact speed.

\subsection{Principle of the method}

The principle of the method is to develop an analytical solution based on models compatible with the studied mechanical phenomena, ensuring the boundary conditions of shear stress on the upper and lower faces.

It is therefore necessary to:

- build a cinematically admissible displacement field satisfying the boundary conditions;

- establish a system of partial differential equations derived from the integration of local equilibrium equations through the thickness to obtain $(u, v, w)$;

- make an energy balance from the formulations based on the displacement field. 


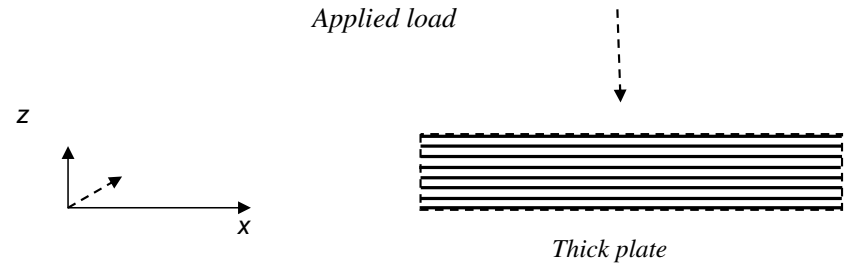

Fig. 2. Composite plate subjected to a transverse load.

\subsection{Dynamic response}

We consider the case of a rectangular orthotropic thick plate simply supported and subjected to a transverse load applied to its mean plane.

The following boundary conditions on the lateral faces of the plate are considered [10]:

$$
\begin{aligned}
& \text { For }\left\{\begin{array} { l } 
{ x = - \frac { a } { 2 } } \\
{ x = \frac { a } { 2 } }
\end{array} \quad \left\{\begin{array}{l}
w=0 \\
\varphi_{y}=0 \\
M_{x}=0
\end{array}\right.\right. \\
& \text { For }\left\{\begin{array} { l } 
{ y = - \frac { b } { 2 } } \\
{ y = \frac { b } { 2 } }
\end{array} \quad \left\{\begin{array}{l}
w=0 \\
\varphi_{x}=0 \\
M_{y}=0
\end{array}\right.\right.
\end{aligned}
$$

An elementary load applied to the upper face $\frac{h}{2}$ is considered following the boundary conditions given in Equations (1) and (2)

$$
\begin{aligned}
& \sigma_{x z}\left(x, y, \pm \frac{h}{2}, t\right)=\sigma_{y z}\left(x, y, \pm \frac{h}{2}, t\right)=0 \\
& \sigma_{z z}\left(x, y, \frac{h}{2}, t\right)=p^{+}(x, y, t) \\
& \sigma_{z z}\left(x, y,-\frac{h}{2}, t\right)=0
\end{aligned}
$$

\subsection{Calculation of the transverse displacement of the plate}

The dynamic response, of an orthotropic symmetrical rectangular plate subject to a concentrated transverse load at a point $\left(x_{0}, y_{0}\right)$, is determined from a modal decomposition. Vertical displacements and external mechanical stresses are expressed in the form of double Fourier series under the assumption of a common base space

$$
\left[\begin{array}{l}
w(x, y, t) \\
p(x, y, t)
\end{array}\right]=\sum_{1}^{\infty} \sum_{1}^{\infty}\left[\begin{array}{l}
q_{n m}(t) g_{n m}^{3}(x, y) \\
p_{n m}(t) g_{n m}^{3}(x, y)
\end{array}\right]
$$

Normal rotations can be expressed in function of two other bases

$$
\varphi_{\beta}(x, y, t)=\sum_{n=1}^{\infty} \sum_{m=1}^{\infty} a_{n m}^{\beta}(t) g_{n m}^{\beta}(x, y)
$$

The $g_{n m}^{i}(x, y), i=1,2,3$ are known functions which satisfy the boundary conditions.
The modal decomposition of the external force is used to identify the coefficients $p_{n m}(t)$. It remains to be identified $a_{n m}^{1}(t), a_{n m}^{2}(t)$ and $q_{n m}(t)$. These parameters can be calculated from the equilibrium Equations (8). To do this, we use the Lagrange equation of equilibrium

$$
L=E_{C}-V_{d} \text { and } \quad \frac{\mathrm{d}}{\mathrm{d} t} \frac{\partial L}{\partial \dot{q}_{n m}}-\frac{\partial L}{\partial q_{n m}}=0
$$

where $V_{d}$ and $E_{c}$ are respectively the potential and the kinetic energies. $V_{d}$ and $E_{c}$ are given by the following expressions

$$
\text { - } E_{C}=\frac{1}{2} \int_{A} \rho_{S}\left(\frac{\partial w}{\partial t}\right)^{2} \mathrm{~d} x \mathrm{~d} y=\sum_{n} \sum_{m} M_{n m} \dot{q}_{n m}^{2}
$$

where $M_{n m}$ is the generalized modal mass with

$$
M_{n m}=\rho_{S} \int_{0}^{a} \int_{0}^{b}\left(g_{n m}^{3}\right)^{2} \mathrm{~d} x \mathrm{~d} y
$$

- $V_{d}=U_{d}-T_{i}$

where $U_{d}=\frac{1}{2} \int_{V} \sigma^{T} \varepsilon d V$ is the energy of deformation and $T_{i}=\int_{A} p(x, y, t) w(x, y, t) \mathrm{d} x \mathrm{~d} y$ is the work of the force of impact.

We solve the problem of a concentrated load

$$
p(x, y, t)=F(t) \delta\left(x-x_{0}\right) \delta\left(y-y_{0}\right)
$$

Lagrange's theorem leads to a differential equation of second order

$$
\ddot{q}_{n m}+2 \xi_{n m} \omega_{n m} \dot{q}_{n m}=\frac{g_{n m}^{3}\left(x_{0}, y_{0}\right) F(t)}{M_{n m}}
$$

whose solution is

$$
\begin{aligned}
q_{n m}(t)= & \frac{g_{n m}^{3}\left(x_{0}, y_{0}\right)}{\varpi_{n m} M_{n m}} \int_{0}^{t} \mathrm{e}^{\left(-\xi_{n m} \omega_{n m}(t-\tau)\right)} \\
& \times F(\tau) \sin \left(\varpi_{n m}(t-\tau)\right) \mathrm{d} \tau
\end{aligned}
$$

$\varpi_{n m}$ is the damped pulse with $\varpi_{n m}=\omega_{n m} \sqrt{1-\xi_{n m}^{2}}$.

$\xi_{n m}$ is critical damping coefficient, which takes into account both the elasticity of the material and the nonlinearity of the supports.

Accordingly, by referring the expression (17) in Equation (13), the response of the plate by modal superposition is reconstructed. The transverse displacement of the plate is given by

$$
\begin{aligned}
& w(x, y, t)=\sum_{n=1}^{\infty} \sum_{m=1}^{\infty} \frac{g_{n m}^{3}\left(x_{0}, y_{0}\right)}{\varpi_{n m} M_{n m}} \\
& \quad \times \int_{0}^{t} \mathrm{e}^{\left(-\xi_{n m} \omega_{n m}(t-\tau)\right)} F(\tau) \sin \left(\varpi_{n m}(t-\tau)\right) \mathrm{d} \tau
\end{aligned}
$$

where the natural frequencies can be determined experimentally or calculated from the fundamental equations of the thick monolithic plate. 
For the determination of natural frequencies, the following characteristic polynomial is used:

$$
\operatorname{det}\left[K_{n m}-\omega_{n m}^{2} M_{n m}\right]=0
$$

where the elements of the matrices are given in the Appendix.

\subsection{Application}

In the case of a thick monolithic plate simply supported, the vertical displacement and rotations are expressed by Equations (10) and (11):

$$
\left\{\begin{array}{l}
w(x, y, t)=\sum_{n=1}^{\infty} \sum_{m=1}^{\infty} q_{n m}(t) \sin \left(n \pi \frac{x}{a}\right) \sin \left(m \pi \frac{y}{b}\right) \\
\varphi_{x}(x, y, t)=\sum_{n=1}^{\infty} \sum_{m=1}^{\infty} a_{n m}^{1}(t) \cos \left(n \pi \frac{x}{a}\right) \sin \left(m \pi \frac{y}{b}\right) \\
\varphi_{y}(x, y, t)=\sum_{n=1}^{\infty} \sum_{m=1}^{\infty} a_{n m}^{2}(t) \sin \left(n \pi \frac{x}{a}\right) \cos \left(m \pi \frac{y}{b}\right)
\end{array}\right.
$$

where the parameters $a_{n m}^{1}(t)$ and $a_{n m}^{2}(t)$ are given by

$$
\left\{\begin{array}{l}
a_{n m}^{1}(t)=\frac{k_{12} k_{23}-k_{13} k_{22}}{k_{11} k_{22}-k_{12}^{2}} q_{m n}(t) \\
a_{n m}^{2}(t)=\frac{k_{12} k_{13}-k_{23} k_{11}}{k_{11} k_{22}-k_{12}^{2}} q_{m n}(t)
\end{array}\right.
$$

where the terms $k_{i j}$ are developed in the Appendix.

Substituting Equation (21) in Equation (20), the transverse displacement and rotations can be written

$$
\left(\begin{array}{rl}
w(x, y, t)= & \frac{4}{a b \rho_{S}} \\
& \times \sum_{n=1}^{\infty} \sum_{m=1}^{\infty} \sin \left(n \pi \frac{x}{a}\right) \sin \left(m \pi \frac{y}{b}\right) \\
& \times \frac{\sin \left(n \pi \frac{x_{0}}{a}\right) \sin \left(m \pi \frac{y_{0}}{b}\right)}{\varpi_{n m}} \\
\times & \int_{0}^{t} \mathrm{e}^{\left(-\xi_{n m} \omega_{n m}(t-\tau)\right)} F(\tau) \sin \left(\varpi_{n m}(t-\tau)\right) \mathrm{d} \tau \\
\varphi_{x}(x, y, t)= & \sum_{n=1}^{\infty} \sum_{m=1}^{\infty} a_{n m}^{1}(t) \sin \left(n \pi \frac{x}{a}\right) \cos \left(m \pi \frac{y}{b}\right) \\
\varphi_{y}(x, y, t)= & \sum_{n=1}^{\infty} \sum_{m=1}^{\infty} a_{n m}^{2}(t) \cos \left(n \pi \frac{x}{a}\right) \sin \left(m \pi \frac{y}{b}\right)
\end{array}\right.
$$

The natural frequencies are equal to

$$
\begin{aligned}
& \omega_{n m}^{2}=\frac{1}{\rho_{S}} \\
\times & {\left[k_{33}+\frac{k_{13}\left(k_{12} k_{23}-k_{13} k_{22}\right)}{k_{11} k_{22}-k_{12}^{2}}+\frac{k_{23}\left(k_{12} k_{13}-k_{11} k_{23}\right)}{k_{11} k_{22}-k_{12}^{2}}\right] }
\end{aligned}
$$
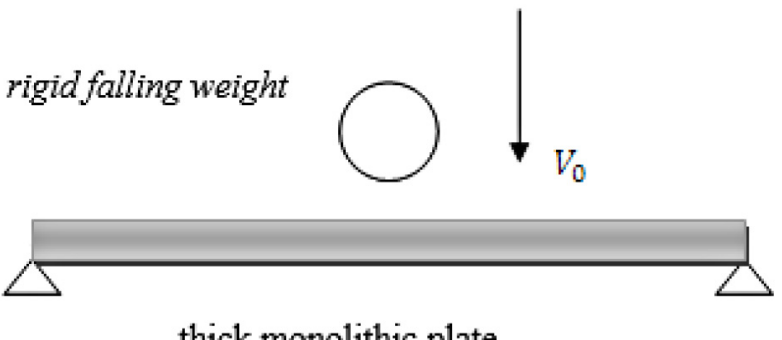

\section{thick monolithic plate}

Fig. 3. Example of configuration of a falling weight.

\subsection{Localized loading}

To simulate shock experiments on falling weight machine, the excitement is assumed to be vertical and located in the center of the plate $\left(x_{0}, y_{0}\right)$. The applied load is developed using a double Fourier series

$$
p(x, y, t)=\sum_{n=1}^{\infty} \sum_{m=1}^{\infty} p_{m n}(t) \sin \left(n \pi \frac{x}{a}\right) \sin \left(m \pi \frac{y}{b}\right)
$$

where

$$
p_{n m}=\frac{4}{a b} \int_{0}^{a} \int_{0}^{b} p_{m n}(t) \sin \left(n \pi \frac{x}{a}\right) \sin \left(m \pi \frac{y}{b}\right)
$$

By substituting $p_{m n}$ by its value in Equation (24), $p(x, y, t)$ (for the case of a localized loading) can be expressed as

$$
\begin{aligned}
p(x, y, t)= & \sum_{n=1}^{\infty} \sum_{m=1}^{\infty} \frac{4 F(t)}{a b} \sin \left(n \pi \frac{x_{0}}{a}\right) \\
& \times \sin \left(m \pi \frac{y_{0}}{b}\right) \sin \left(n \pi \frac{x}{a}\right) \sin \left(m \pi \frac{y}{b}\right)
\end{aligned}
$$

\section{Contact force}

Consider two bodies that will collide, one is elastic and the other rigid. As part of the study of the impact behavior of a thick monolithic plate, the contact is modeled by the Hertz law [Lee 93]

This contact law (sphere on plane), is of the type

$$
F=k_{h}(\alpha)^{3 / 2}
$$

where $F$ is the contact force during impact and $k_{h}$ is the hertzian coefficient which depends on the elastic properties of two bodies. This coefficient can be experimentally determined by a quasi-static loading test. The penetration $\alpha$ is given by

$$
\alpha(t)=s(t)-w\left(x_{0}, y_{0}, t\right)
$$

where $s(t)$ represents the displacement of the impactor, and $w\left(x_{0}, y_{0}, t\right)$ the transverse displacement of the structure at the point of impact. 
M.A. Wahab et al.: Mechanics \& Industry 17, 414 (2016)

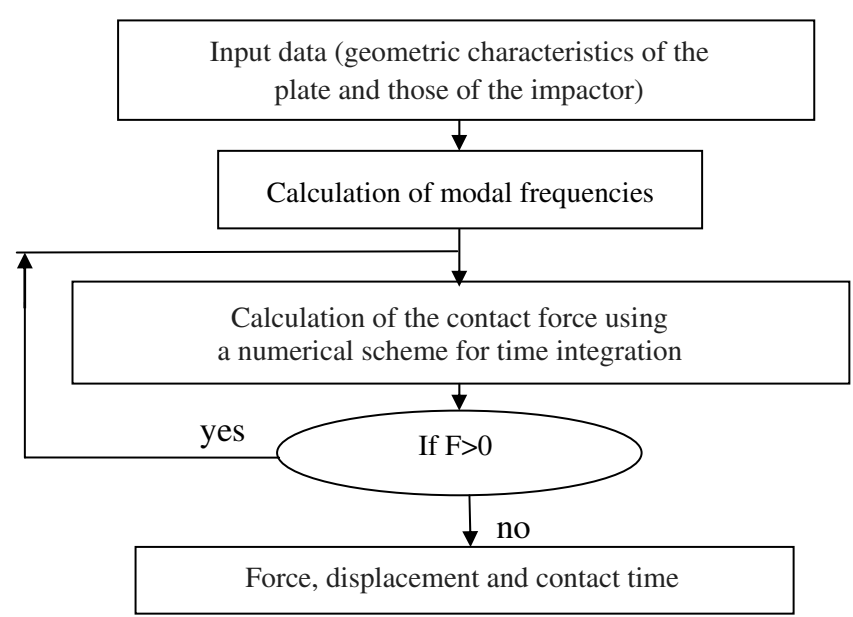

Fig. 4. Shock algorithm using an iterative method. by

The transverse displacement of the impactor is given

$$
s(t)=V_{0} t-\frac{1}{m_{0}} \int_{0}^{t} F(\tau)(\tau-t) \mathrm{d} \tau
$$

where $V_{0}$ is the velocity of the impactor before impact and $m_{0}$ the mass of the impactor.

By referring Equations (18) and (28) in Equation (27), a new expression appears:

$$
\begin{aligned}
&\left(\frac{F}{k_{h}}\right)^{2 / 3}=V_{0} t-\frac{1}{m_{0}} \int_{0}^{t} F(\tau)(\tau-t) \mathrm{d} \tau \\
&-\sum_{n=1}^{\infty} \sum_{m=1}^{\infty} \frac{g_{n m}^{3}\left(x_{0}, y_{0}\right)}{\varpi_{n m} M_{n m}} \\
& \times \int_{0}^{t} \mathrm{e}^{\left(-\xi_{n m} \omega_{n m}(t-\tau)\right)} F(\tau) \sin \left(\varpi_{n m}(t-\tau)\right) \mathrm{d} \tau
\end{aligned}
$$

This nonlinear integral equation obtained can be solved by a numerical method. Reference may be made to the work of Sun and Chattopadhyay [13] using an explicit method with increments of time. Calculating the contact force provides transverse movement of the plate by application of Equation (29). The used algorithm is shown in Figure 4.

We were led to develop numerical simulation of the dynamic response, taking into account the damping in the model. This comes from the viscoelasticity of the material or the loss of energy at the supports (poor quality of the installation, matting at the supports, etc.). In the procedure of calculation of the contact force (see Fig. 4), we use the following algorithm.

The first time step $F_{1}$ is calculated from $V_{0}$.

$$
F_{1}=k_{h}\left(V_{0} \delta \tau\right)^{3 / 2}
$$

At time $i, F_{i}$ is calculated from $V_{(i-1)}$ and $F_{(i-1)}$ :

$$
\begin{aligned}
& \left(F_{i} / k_{h}\right)^{2 / 3}=V_{(i-1)} \delta \tau-\frac{1}{m_{0}} \sum_{j=0}^{i-1} F_{j} \delta \tau^{2} \frac{(2 i-2 j-1}{2} \\
& -\sum_{n} \sum_{m} \frac{4\left(g_{n m}^{3}\left(x_{0}, y_{0}\right)\right)^{2}}{\left(a b \rho h \varpi_{n m}^{2}\right)} \sum_{j=0}^{i-1}\left(f_{1}(j, i)-f_{2}(j, i)\right)
\end{aligned}
$$

Table 1. Characteristics of the plates.

\begin{tabular}{ccc}
\hline Properties & Average value & Obtained by \\
\hline Density & $1600 \mathrm{~kg} / \mathrm{m}^{3}$ & Weighing \\
\hline Young's modulus & $E_{1}=E_{2}=14 \mathrm{GPa}$ & Static test \\
& $E_{3}=8 \mathrm{GPa}$ & $\begin{array}{c}\text { Ultrasonic } \\
\text { measurement }\end{array}$ \\
\hline Shear modulus & $G_{12}=G_{13}=4 \mathrm{GPa}$ & Static test \\
\hline Poisson's ratio & $\nu_{12}=\nu_{32}=0.25$ & Static test \\
\hline Thickness & $14.5 \mathrm{~mm}$ & Average of \\
& & 30 measurements \\
\hline
\end{tabular}

Length of the plate: $a=0.3 \mathrm{~m}$, width of the plate: $b=0.3 \mathrm{~m}$.

where

$$
\begin{aligned}
f_{1}(j, i)= & \mathrm{e}^{\left(-\omega_{n m} \xi_{n m}(i-j-1)\right)} \delta \tau F_{j}\left[\operatorname { c o s } \left(\varpi_{n m}(i-j-1) \delta \tau\right.\right. \\
& +\xi_{n m} \sin \left(\left(\varpi_{n m}(i-j-1) \delta \tau\right]\right. \\
f_{2}(j, i)= & \mathrm{e}^{\left(-\omega_{n m} \xi_{n m}(i-j)\right)} \delta \tau F_{j}\left[\operatorname { c o s } \left(\varpi_{n m}(i-j) \delta \tau\right.\right. \\
& +\xi_{n m} \sin \left(\left(\varpi_{n m}(i-j) \delta \tau\right]\right.
\end{aligned}
$$

The iterations are continued until an $N$ such that $F=0$. So $N d \tau$ is the contact time.

\section{Results}

\subsection{Dynamic analysis of a thick monolithic plate}

We propose to examine in detail the dynamic behavior of a rectangular monolithic plate $(300 \times 300 \times 15 \mathrm{~mm})$ of composite material simply supported on four sides, with 12 identical glass fiber plies (a ply is formed by a layer of tissue $0 / 90$ of $500 \mathrm{~g} / \mathrm{m}^{2}$ and a layer of $300 \mathrm{~g} / \mathrm{m}^{2}$ ) in a polyester resin. This plate is subjected to the impact of a rigid sphere falling from a variable height ranging from $0.25 \mathrm{~m}$ to $2 \mathrm{~m}$. The geometrical characteristics of the plate and the material properties are given in Table 1.

The steel impactor properties are: mass $m_{0}=10.9 \mathrm{~kg}$, radius: $R=50 \mathrm{~mm}$. The coefficient of penetration is obtained by a quasi-static loading test: $k_{h}=2.2 \times$ $10^{8} \mathrm{~N} / \mathrm{m}^{1.5}$.

Figures 5 and 6 show that the results obtained with the approach developed here are similar to those obtained by the experiment ( $10 \%$ difference of the force and displacement). Better correlation could be obtained by varying parameters such as stiffness crushing from these trials.

The comparative study of Tables 2 and 3 shows that the calculation results are close to the measured values. For displacement, the error is of the order of $5 \%$.

\section{Conclusion}

The purpose of this work was to develop a simple analytical model for the simulation of the dynamic response of a structure subjected to a low-speed impact, to predict 


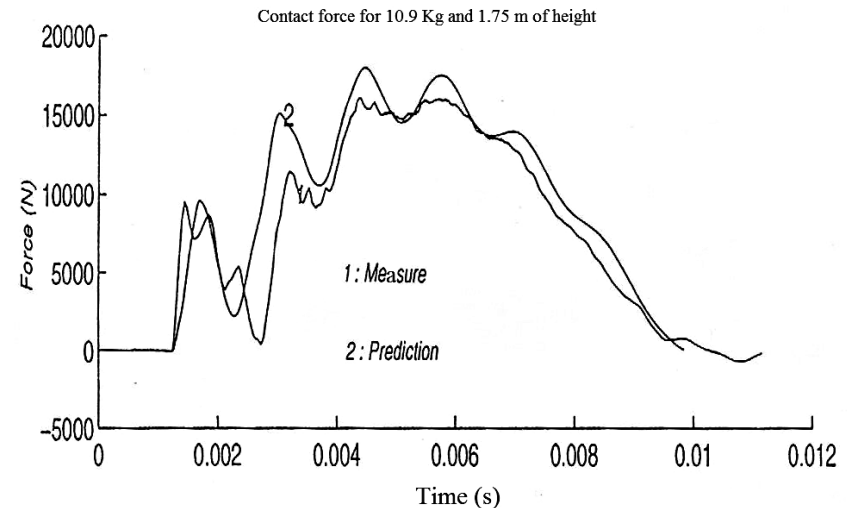

Fig. 5. Comparison between theoretical and experimental results of the force of contact.

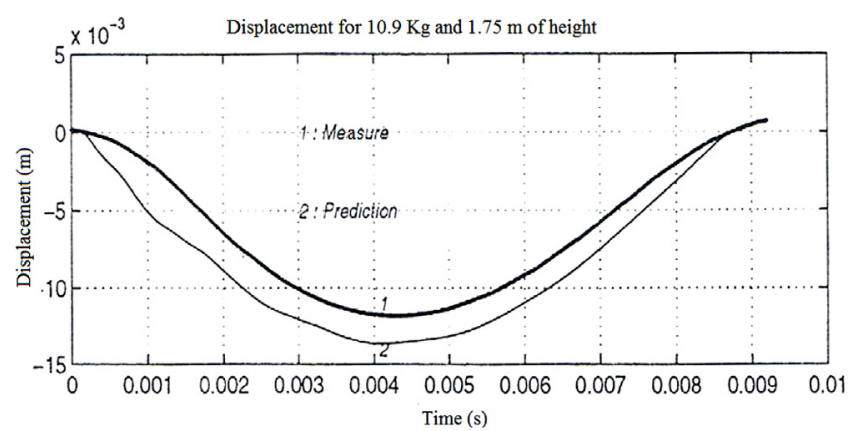

Fig. 6. Displacement of the thick plate with time obtained by experiment and by the analytical method by modal superposition with 4 modes taken into account in the calculation for a damping $\xi_{n m}=10 \%$.

Table 2. Contact force $\left(\times 10^{4}(N)\right)$ at the center of the thick plate for $10.9 \mathrm{~kg}$.

\begin{tabular}{cccc}
\hline Height $(\mathrm{m})$ & 1 & 1.5 & 1.75 \\
\hline Prediction/measuring & $1.7 / 1.8$ & $2.15 / 1.95$ & $2.33 / 2.50$ \\
relative error & $-5.8 \%$ & $9.3 \%$ & $-7.2 \%$ \\
\hline
\end{tabular}

the initiation of a global damage. This model was developed from the theory of anisotropic elastic thick plates based on an approach which takes into account the transverse shear.

The dynamic modal acceleration approach allows very significantly reduction of the number of modes to be considered by first integrating the static solution.

The developed method was validated by a double comparison, experiments with falling weight machines and with a semi-analytical energy model. The results are in good agreement with regard to the vertical displacement, the contact force and the duration of contact. These comparisons have highlighted the importance of damping due to boundary conditions at supports.

The result of our work can be used to predict the transverse shear deformation in the core of a sandwich plate to apply a failure criterion that will predict the occurrence of damage due to impact.
Table 3. Displacement $(\mathrm{mm})$ at the center of the thick plate for $10.9 \mathrm{~kg}$.

\begin{tabular}{cccc}
\hline Height $(\mathrm{m})$ & 1 & 1.5 & 1.75 \\
\hline Prediction/measuring & $9.5 / 9.9$ & $12.5 / 11.5$ & $13 / 11.8$ \\
relative error & $-4.2 \%$ & $8 \%$ & $9.2 \%$ \\
\hline
\end{tabular}

\section{Appendix}

The elements of the stiffness matrix $(9,12)$ are defined, as following:

$$
\begin{aligned}
k_{11}= & D_{11} \alpha_{1}^{2}+D_{66} \beta_{1}^{2}+A_{55}-\frac{4}{h^{2}} D_{55} \\
& -\frac{4}{h^{2}}\left(F_{11} \alpha_{1}^{2}+F_{66} \beta_{1}^{2}\right)-\left(\overline{D_{55}}-\frac{4}{3 h^{2}} \overline{F_{55}}\right) \alpha_{1}^{2} \\
k_{12}= & \left(D_{12} \alpha_{1}^{2}+D_{66} \beta_{1}^{2}+A_{55}-\frac{4}{3 h^{2}}\left(F_{11}-F_{66}\right)\right. \\
& \left.-\frac{4}{3 h^{2}}\left(F_{11}-F_{66}\right)-\left(\bar{D}_{55}-\frac{4}{3 h^{2}} \bar{F}_{55}\right)\right) \alpha_{1} \beta_{1} \\
k_{13}= & \left(A_{55}-\frac{4}{h^{2}} D_{55}\right) \alpha_{1} \\
& \left.-\frac{4}{3 h^{2}}\left[F_{11} \alpha_{1}^{3}-\left(F_{11}+2 F_{66}\right)-F_{66}\right) \alpha_{1} \beta_{1}^{2}\right] \\
k_{22}= & D_{22} \beta_{1}^{2}+D_{66} \alpha_{1}^{2}+A_{55}-\frac{4}{h^{2}} D_{44} \\
& -\frac{4}{h^{2}}\left(F_{22} \beta_{1}^{2}+F_{66} \alpha_{1}^{2}\right)-\left(\bar{D}_{44}-\frac{4}{3 h^{2}} \bar{F}_{44}\right) \beta_{1}^{2} \\
k_{23}= & \left(A_{44}-\frac{4}{h^{2}} D_{44}\right) \beta_{1}-\frac{4}{3 h^{2}} \\
& \left.\times\left[F_{22} \beta_{1}^{3}-\left(F_{12}+2 F_{66}\right)-F_{66}\right) \beta_{1} \alpha_{1}^{2}\right] \\
& -\left(\bar{D}_{44}-\frac{4}{3 h^{2}} D_{44}\right) \beta_{1}^{3}-\left(\bar{D}_{55}-\frac{4}{3 h^{2}} \bar{F}_{55}\right) \alpha_{1} \beta_{1}^{2} \\
k_{33}= & \left(A_{55}-\frac{4}{h^{2}} D_{55}\right) \alpha_{1}^{2}-\left(A_{44}-\frac{4}{h^{2}} D_{44}\right) \beta_{1}^{2} \\
& \left({ }^{2}\right)
\end{aligned}
$$

With $\alpha_{1}=n \frac{\pi}{a}$ and $\beta_{1}=m \frac{\pi}{a}$

$$
\begin{aligned}
A_{i j}, D_{i j}= & \int_{-h / 2}^{h / 2} Q_{i j}\left(1, z^{2}\right) \mathrm{d} z \text { for } i, j=1 \text { to } 6 \\
F_{i j}= & \int_{-h / 2}^{h / 2} Q_{i j}\left(z^{4}\right) \mathrm{d} z \text { for } i, j=1,2,6 \\
\bar{D}_{44}, \bar{F}_{44}= & \int_{-h / 2}^{h / 2} Q_{44} R_{1}\left(z^{2}, z^{4}\right) \mathrm{d} z \\
\bar{D}_{55}, \bar{F}_{55}= & \int_{-h / 2}^{h / 2} Q_{55} R_{2}\left(z^{2}, z^{4}\right) \mathrm{d} z
\end{aligned}
$$


M.A. Wahab et al.: Mechanics \& Industry 17, 414 (2016)

\section{References}

[1] R. Hosseinzadeh, M. Shokrieh, L. Lessard, Damage behavior of fiber reinforced composite plates subjected to drop weight impacts, Composites Sci. Technol. 66 (2006) 61-68

[2] G. Perillo, N.P. Vedivik, A.T. Echtermeyer, Damage development in stitch bonded GFRP composite plates under low velocity impact: Experimental and numerical results, J. Composite Mater. 49 (2015) 601-615

[3] F. Caputo, A. De Luca, G. Lamanna, R. Borrelli, U. Mercurio, Numerical study for the structural analysis of composite laminates subjected to low velocity impact, Composites B, Eng. 67 (2014) 296-302

[4] Z. Aslan, R. Karakuzu, B. Okutan, The response of laminated composite plates under low-velocity impact loading, Composite Struct. 59 (2003) 119-128

[5] I. Guiamatsia, J.K. Ankersen, L. Iannucci, M. Fouinneteau, Enriched finite elements for the efficient prediction of impact-induced damage in composite laminates, Composites Sci. Technol. 79 (2013) 87-96

[6] N. Goo, K. Seo, J. Seung, Dynamic contact analysis of laminated composite plates under low-velocity impact, AIAA J. 35 (1997) 1518-1524

[7] G. Reyes, U. Sharma, Modeling and damage repair of woven thermoplastic composites subjected to low velocity impact, Composite Struct. 92 (2010) 523-531
[8] J.N. Reddy, A refined Non-linear theory of plates with Tranverse Shear Deformation, Int. J. Solids Struct. 20 (1998) 881-899

[9] G. Cederbaum et al., Random Vibration and Reliability of Composites structures, Technomic Publication Company Co. Inc., 1992, pp. 28-53

[10] J.M. Berthelot, Matériaux composites. Comportement mécanique et analyse des structures, Collection Masson, 1998

[11] W.C. Jackson, C. Jr Poe, The Use of impact Force as a Scale Parameter for the impact response of Composite laminates, J. Composites Technol. Res. 15 (1993) 282-289

[12] S. Thangjithan, L. Librescu, G. Cederbaum, LowVelocity Impact Response of Orthotropic plates Using a Higher-Order Theory, AIAA/ASME/ASCE/AHS 48th Structures, Structural Dynamics and Materials Conference, Monterey, CA, April 6-8, 1987, pp. 448-457

[13] C.T. Sun, S. Chattopadhyay, Dynamic response of anisotropic laminated plates under initial stress ti impact mass, J. Applied Mech. 42 (1975) 693-698 